\title{
Estimação da área foliar da "jitirana" (Merremia aegyptia (1.) Urban), através de modelos de regressão para Mossoró - RN
}

\section{Estimation of leaf area "scarlet starglory" (Merremia aegyptia (1.) Urban) through regression models}

\section{Janilson Pinheiro de Assis ${ }^{1}$, Paulo César Ferreira Linhares ${ }^{2}$, Roberto Pequeno de Souza ${ }^{3}$, Maria Francisca Soares Pereira ${ }^{4}$ Alany Moisa Bezerra de Almeida ${ }^{5}$}

RESUMO - A mensuração da área foliar é requerida em vários estudos agronômicos, ecológicos e biológicos de uma maneira geral. O objetivo deste trabalho foi obter equações de regressões lineares e não lineares que estimem a área foliar real da espécie jitirana, em função das dimensões do comprimento ao longo da nervura principal (C) e largura máxima (L). Para isso 200 limbos foliares foram coletados em ecossistemas de ocorrência natural desta espécie vegetal, na região de Mossoró, no Estado do Rio Grande do Norte, limpas e acondicionadas em caixa de isopor e medidas imediatamente, inclusive sua área real através do Integrador de Área foliar. Considerando a parcimônia do modelo, o coeficiente de determinação e a significância do teste $\mathrm{F}$ da análise de variância a $3 \%$ de probabilidade, as melhores equações para estimação da área foliar da jitirana em ordem de importância foram: modelo linear simples passando pela origem em função do produto do comprimento com a largura da folha; linear simples em função do comprimento; linear simples em função da largura; e o modelo de regressão linear múltipla, modelada em função do comprimento e largura simultaneamente. Onde Merremia aegyptia apresentou valores médios de comprimento das folhas, largura e área foliar real iguais a $13,5 \mathrm{~cm}, 28,8 \mathrm{~cm}$ e 202,38 $\mathrm{cm}^{2}$, respectivamente. $95 \%$ da área foliar de 200 limbos está relacionada com folhas de tamanho variando de $133,3 \mathrm{~cm}^{2}$ a $299,0 \mathrm{~cm}^{2}$.

Palavras - chave: Mensuração foliar. Convolvulaceae. Modelagem.

\begin{abstract}
The measurement of leaf area is required in several agronomic studies, ecological and biological processes in general. The objective of this study was to obtain equations of linear and nonlinear regressions to estimate the real leaf area of Scarlet Starglory, depending on the dimensions of length along the main vein (C) and width (L). For this 200 leaves were collected in areas of naturally occurring ecosystems of this plant species in the region of Mossoró, in Rio Grande do Norte state, Brazil, the plants were cleaned and wrapped, then they were measured immediately through Integrador of Area to foliate. Considering the parsimony of the model, the coefficient of determination, and the significance of the $\mathrm{F}$ test of analysis of variance of $3 \%$ probability, best equations that estimated the leaf area were in order of importance was: the model lineal simple going by the origin in function of the product of the length with the width of the leaf; lineal simple in function of the length; lineal simple in function of the width; and the model of multiple lineal regression, modeled in function of the length and width simultaneously. Where Merremia aegyptia apresentau medium values of length of the leaves, width and area to foliate real iguias to $13.5 \mathrm{~cm}, 28.8 \mathrm{~cm}$ and $202.38 \mathrm{~cm}^{2}$, respectively.
\end{abstract}

Keywords: Leaf mensuration. Convolvulaceae. Model.

\footnotetext{
*Autor para correspondência

Recebido para publicação em 14/08/2015; aprovado em 20/11/2015

${ }^{1}$ Engenheiro Agrônomo, Doutor em Agronomia- Professor da Universidade Federal Rural do Semi-Árido (UFERSA) E-mail: janilson@ufersa.edu.br.

${ }^{2}$ Eng $^{\circ} \quad$ Agr $^{\circ}$ DSc., Paulo César Ferreira Linhares. PESQUISADOR DA UFERSA, Mossoró-RN. E-mail:

paulolinhares@ufersa.edu.br;paulojitirana@yahoo.com.br

${ }^{3}$ Engenheiro Agrícola, Doutor em Agronomia- Professor da Universidade Federal Rural do Semi-Árido (UFERSA) E-mail: rpequeno@ufersa.eu.br

${ }^{4}$ Eng $^{\mathrm{a}}$ Agr $^{\mathrm{a}}$ DSc. Maria Francisca Soares Pereira. GRUPO VERDE, POMBAL-PB. E-mail: mf.agro@ yahoo.com.br

${ }^{5}$ Mestranda em Sistemas Agroindustriais CCTA-Pombal, Alany Moisa Bezerra de Almeida. UFCG. E-mail:alanymoisa1@hotmail.com
} 


\section{INTRODUÇÃO}

A jitirana é uma espécie pertencente à família das convolvuláceas considerada uma espécie espontânea do bioma caatinga, uma liana (hábito trepador), anual, herbácea, com produção média de fitomassa verde e seca da ordem de 36000 e $4000 \mathrm{~kg} \mathrm{ha}^{-1}$, respectivamente, com teor de nitrogênio de $26,2 \mathrm{gkg}^{-1}$ na matéria seca (LINHARES et al., 2008), possui relação $\mathrm{C} / \mathrm{N}$ de $18 / 1$, o que viabiliza a espécie para uso como adubo verde pela rápida decomposição da palhada (LINHARES et al., 2012).

Normalmente, existe interesse por métodos de mensuração simples, rápidos e que não destruam a folha. $\mathrm{O}$ conhecimento da área foliar é fundamental no estudo do desenvolvimento das plantas, sendo talvez o mais importante parâmetro (BIANCO; PITELLI; PERECIN, 1983). O primeiro passo para se estudar o crescimento das plantas é conhecer características do crescimento e desenvolvimento da planta. Dentre essas características, o conhecimento da área foliar é um importante parâmetro para o entendimento da fotossíntese, interceptação luminosa, uso da água e nutrientes e o potencial produtivo. Neste contexto, torna-se fundamental o conhecimento da área foliar para ajudar o entendimento do processo de partição de assimilados e na determinação do número de folhas ideal nas condições climáticas do Brasil. Uma vez que área foliar é uma característica difícil de ser mensurada, pois requer equipamentos caros ou técnicas destrutivas, torna-se muito importante à determinação de uma equação que possa estimar a área foliar das plantas a partir de valores, que podem ser obtidos de forma não destrutiva.

Existem vários métodos para se medir a área foliar com boa precisão, sendo classificados em destrutivos e não destrutivos, diretos ou indiretos (MARSHALL, 1968).

A importância de utilizar um método não destrutivo é que ele permite acompanhar o crescimento e a expansão foliar da mesma planta até o final do ciclo ou do ensaio, além de ser rápido e preciso. Assim, a área foliar pode ser estimada utilizando-se parâmetros dimensionais de folhas, os quais apresentam boas correlações com a superfície foliar. Um dos métodos não destrutivos mais utilizados é a estimativa da área foliar por meio de equações de regressão entre a área foliar real (Sf) e os parâmetros dimensionais lineares das folhas. Este método já foi utilizado com sucesso para plantas cultivadas, como abóbora (SILVA et al., 1998), videira em cultivar Niagara rosada (PEDRO JÚNIOR; RIBEIRO; MARTINS, 1986) e para plantas daninhas, como Wissadula subpeltata (BIANCO; PITELLI; PERECIN, 1983), Senna obtusifolia (PERESSIN; PITELLI; PERECIN, 1984), Amaranthus retroflexus (BIANCO et al., 1995), Richardia brasiliensis (ROSSETO; PITELLI, 1997), Solanum americanum (TOFOLI et al., 1998), Cissampelos glaberrima (BIANCO; PITELLI; CARVALHO, 2002) e Typha latifolia (BIANCO; PITELLI , 2003).

Souza Neto (2009) desenvolveu um estudo comparativo entre diferentes procedimentos de cálculo da área foliar, de cinco espécies florestais ocorrentes no semiárido paraibano, considerando como referência valores de área foliar obtido por processo de digitalização de imagens. A partir de medidas do comprimento ao longo da nervura principal (C) e da largura máxima $(\mathrm{L})$, perpendicular à nervura principal, foram calculadas as áreas foliares pela equação da elipse e pelos modelos linear e potencial. Os resultados mostraram que todos os modelos avaliados (elíptico, linear e potencial) podem ser usados para estimativa da área foliar das cinco espécies estudadas. $\mathrm{O}$ uso do modelo potencial permite estimar a área foliar da espécie Craibeira com maior nível de precisão. Souza Lima et al. (2008), concluíram que medidas de área foliar de feijão caupi podem ser estimadas a partir de modelos potencial e linear com boa precisão, utilizando equações envolvendo medidas de comprimento e largura.

Silva et al. (2002), estudando as estimativas de área de folhas de plantas de gergelim verificaram que essas estimativas são mais precisas quando se usam ambas as dimensões, ou seja, comprimento e largura do limbo, e obtiveram as equações $S=4,0438+0,6075 \mathrm{CL}, \mathrm{S}=0,631 \mathrm{CL}$ e $\mathrm{S}=0,70 \mathrm{CL}$, como as que tiveram melhor ajuste ao modelo linear, com $\mathrm{r}^{2}$ igual a 0,9746, 0,9857 e 0,9872, respectivamente.

Bianco, Carvalho e Bianco (2008), utilizaram os modelos estatísticos linear: $\mathrm{Y}=\mathrm{a}+\mathrm{bx}$; linear simples: $\mathrm{Y}=$ bx; geométrico: $\mathrm{Y}=\mathrm{ax}^{\mathrm{b}}$; e exponencial: $\mathrm{Y}=\mathrm{ab}^{\mathrm{x}}$, e concluíram que todos os modelos analisados podem ser empregados para estimação da área foliar de S. cordifolia e S. rhombifolia. No entanto eles sugerem optar pela equação linear simples, envolvendo o produto $\mathrm{C} * \mathrm{~L}$, considerando-se o coeficiente linear igual a zero, em função da praticidade desta. Desse modo, a estimativa da área foliar de $S$. cordifolia foi obtida pela fórmula Af $=0,7878(\mathrm{C} * \mathrm{~L})$, com coeficiente de determinação de 0,9307 , enquanto para $S$. rhombifolia a estimativa da área foliar foi obtida pela fórmula Af = 0,6423(C*L), com coeficiente de determinação de 0,9711 .

Duarte et al. (2009), também utilizaram os modelos estatísticos linear simples, $Y=a+b x$; linear simples passando pela origem, $\mathrm{Y}=\mathrm{bx}$; geométrico, $\mathrm{Y}=\mathrm{ax}{ }^{\mathrm{b}}$; e exponencial, $\mathrm{Y}=$ $\mathrm{ab}^{\mathrm{x}}$, e verificaram também que todos os modelos analisados podem ser empregados na estimação da área foliar de $E$. heterophylla. Neste caso sugeriram optar pela equação linear simples envolvendo $\mathrm{C} * \mathrm{~L}$, considerando o coeficiente linear igual a zero, em razão de sua praticidade. Desse modo, a estimativa da área foliar de E. heterophylla foi obtida pela equação Af' = 0,6816(C*L).

Bianco et al. (2007), afirmaram que a estimativa da área foliar de I. hederifolia pode ser feita pela fórmula $\mathrm{Sf}=0,7583$ $\mathrm{x}(\mathrm{C} \times \mathrm{L})$, ou seja, $75,83 \%$ do produto entre o comprimento ao longo da nervura principal e a largura máxima, ao passo que, para I. nil, a estimativa da área foliar pode ser feita pela fórmula $\mathrm{Sf}=0,6122\left(\mathrm{C}^{*} \mathrm{~L}\right)$, ou seja, $61,22 \%$ do produto entre o comprimento ao longo da nervura principal e a largura máxima da folha.

Pinto et al. (2007), sugeriram que do ponto de vista prático, pode-se optar pela equação linear simples, considerando como fator de correção o valor do coeficiente angular obtido da relação entre o produto $\mathrm{C} * \mathrm{~L}$ e a área foliar real, em que a reta passa pela origem, ou seja, $a=0$. Dessa forma a estimativa da área foliar da maniçoba independente da espécie, pode ser feita pela equação $\mathrm{AF}=\left(\mathrm{C}^{*} \mathrm{~L}\right) \times 0,533$ sendo o coeficiente de determinação de 0,89 .

Convém salientar que a exigência de que os valores da variável independente $\mathrm{X}$ sejam fixados, isto é controladas pelo experimentador ou por outras pessoas ou instituição em amostras repetidas só tem interesse teórico, pois, na realidade, raramente se retiram ou observam-se mais de uma amostra para determinado conjunto de valores de X. O propósito dessa exigência é estabelecer a modalidade para as distribuições 
amostrais dos vários estimadores dos parâmetros da equação de regressão. Isto realmente equivale a dizer que se retirarmos um número infinito de amostras de um tamanho, isto, é, um número infinito de conjuntos de valores de $\mathrm{Y}_{1}, \mathrm{Y}_{2}, \ldots, \mathrm{Y}_{\mathrm{n}}$ para um conjunto fixo de valores de $X$, ou seja, um conjunto de valores de $\mathrm{X}_{1}, \mathrm{X}_{2}, \ldots \mathrm{X}_{\mathrm{n}}$, então as distribuições amostrais e as propriedades dos estimadores serão as desejáveis de um bom estimador. Assim evita-se que complicações possam surgir se os valores de $\mathrm{X}$ tivessem de mudar de amostra para amostra. Vale lembrar que os valores de $\mathrm{X}$ podem ser considerados fixos de amostra para amostras mesmo que $X$ seja uma variável estocástica, enquanto que $\mathrm{X}$ sendo fixo não faz com que Y seja fixo também (KMENTA, 1990).

O aspecto trabalhoso da estimação através do método da Máxima Verossimilhança para modelos de regressão não linear, o qual é um método não linear, tem como característica a descoberta real das estimativas dos parâmetros $\theta$ 's, onde essas estimativas são obtidas por meio de um computador graças à aplicação sistemática do método de tentativa e erro, ou seja, métodos numéricos, como o de Gauss Newton, Newton - Rapson, etc., que exigem um valor inicial para o parâmetro $\theta$. Neste caso utiliza-se uma técnica matemática onde a série chama-se expansão de $\mathrm{f}(\mathrm{x})$ da série de Taylor no ponto $\mathrm{x}=\mathrm{a}$. Em essência, o que o computador faz é substituir diferentes valores dos parâmetros na função de verossimilhança, e assim calcular o valor da função e repetir esses passos até que não possa haver mais aumento no valor da função Verossimilhança, e por isso o valor do seu logaritmo deve ser o maior possível com sinal negativo. Tal processo dá ótimos resultados se a função de Verossimilhança só tiver um pico bem definido, mas pode haver problemas se essa função tiver mais de um pico ou se ela for muito achatada no topo, onde nesta situação a função de Veromilhança é muito sensível a mudanças nos dados amostrais da série histórica, o que significa que mesmo um leve erro de medida ou um erro de arredondamento pode fazer oscilar, de maneira marcante o valor do parâmetro teta $(\theta)$ e eliminar, assim o, alto grau de confiança, na estimativa resultante. Isso ocorre, porque se o computador iniciar sua pesquisa para maximização do valor do parâmetro $\theta$, na vizinhança de $\hat{\boldsymbol{\theta}}_{\mathbf{1}}$, ele irá parar em $\hat{\boldsymbol{\theta}}_{\mathbf{1}}$ e apresentará este resultado como a estimativa de Máxima Verossimilhança de $\theta$, enquanto a estimação correta de Máxima Verossimilhança de $\theta$ é, na verdade $\hat{\boldsymbol{\theta}}_{\mathbf{2}}$. Não há muito que fazer, neste caso, salvo fazer com que o computador estude todo o domínio dos valores possíveis de $\theta$, no entanto se tal domínio for infinito o computador estará diante de uma tarefa que não pode ser executada. Frequentemente, porém, o domínio dos valores paramétricos é finito e, neste caso, o problema de se achar o ponto de máximo absoluto torna-se possível (KMENTA, 1990).

Diante do exposto este trabalho foi realizado com o objetivo de avaliar o poder explicativo de diferentes modelos de equações de regressão lineares e não lineares para a determinação da área foliar da jitirana em função do seu comprimento ao longo da nervura principal e de sua maior largura.

\section{MATERIAL E MÉTODOS}

O experimento foi conduzido no campus da Universidade Federal Rural do Semi-Árido (UFERSA),

localizada na cidade de Mossoró, Estado do Rio Grande do Norte. A região de Mossoró de acordo com Carmo Filho (1991) apresenta precipitação anual em torno de $67 \mathrm{~mm}$, com temperatura média de $27,40^{\circ} \mathrm{C}$, latitude sul $5^{\circ} 11$ '; longitude oeste $37^{\circ} 20^{\prime}$; umidade relativa do ar, $68,90 \%$, velocidade do vento $4,10 \mathrm{~m} / \mathrm{s}$; altitude ao nível do mar $18 \mathrm{~m}$; com ventos predominantes no sentido nordeste; pressão atmosférica $757,30 \mathrm{mmHg}$; insolação 236h/mês; evaporação a sombra $5,75 \mathrm{~mm} /$ dia e evaporação a céu aberto $7,70 \mathrm{~mm} /$ dia.

Foram coletados um total de 200 limbos foliares de jitirana (Merremia aegyptia), em ecossistemas de ocorrência natural desta espécie vegetal, sujeitos às mais diferentes condições ecológicas em que a espécie pode ocorrer, considerando todas as folhas das plantas, desde que não apresentassem deformações oriundas de fatores externos, como pragas, doenças e efeitos de fatores meteorológicos. Limpas e acondicionadas em caixa de isopor e medidas imediatamente, após a chegada em Laboratório para estimação da área real. Nesse procedimento procurou-se evitar que a perda de turgidez das folhas pudesse alterar os resultados.

$\mathrm{Na}$ fase de obtenção dos dados no campo, foram coletadas a esmo de 10 a 25 folhas em cada planta, as quais foram levadas ao laboratório, para a determinação do comprimento do limbo foliar ao longo da nervura principal (C) e da largura máxima do limbo foliar (L) perpendicular à nervura principal. Em seguida suas áreas foliares reais (AR) foram determinadas com a utilização do aparelho Portable Área Meter Licor Mod. L1 - 3000 (testemunha).

No caso do presente estudo, as variáveis independentes $\mathrm{X}$ (comprimento e largura) foram consideradas fixas e a dependente Y (área foliar) foi admitida aleatória.

Para selecionar um modelo de equação que pudesse representar a área foliar em função das dimensões foliares comprimento e largura, procedeu-se a estudos de ajustes de modelos de regressão, utilizando as seguintes equações: linear simples (eq. 1), linear simples passando pela origem, ajustando apenas para o produto entre comprimento largura (eq. 2), geométrica (eq. 3), exponencial (eq. 4), logarítmica (eq. 5) e linear múltipla (eq. 6). O valor de y estimou a área foliar do limbo foliar em função de $\mathrm{X}$, cujos valores podem ser o comprimento $(\mathrm{C})$, a largura $(\mathrm{L})$, o produto $(\mathrm{C} \times \mathrm{L})$ ou a soma $(\mathrm{C}+\mathrm{L})$.

$$
\mathbf{Y}=\mathbf{a}+\mathbf{b} \mathbf{X}
$$

$$
\mathbf{Y}=\mathbf{b X}
$$

$$
Y=a e^{(b x)}
$$

$$
\mathbf{Y}=\mathbf{a} \mathbf{X}^{\mathbf{b}}
$$

$$
\mathbf{Y}=\mathbf{a}+\mathbf{b} \cdot \ln (\mathrm{X})
$$

$$
Y=a+b_{1} X_{1}+b_{2} X_{2}
$$


$\mathrm{O}$ valor $\mathrm{Y}$ estima a área real em função de $\mathrm{x}$, cujos valores podem ser o comprimento $(C)$, a largura $(L)$ o produto $(C \times L)$, ou a soma $(\mathrm{C}+\mathrm{L})$. No caso de $\mathrm{x}=C \times L$, Estimou-se também a equação linear passando pela origem, o que praticamente significa supor que a área é proporcional a um retângulo. A equação a ser escolhida será a que apresentar a menor soma de quadrados do resíduo (menor valor $\mathrm{p}$ do teste F de Fisher-Snedecor, maior valor para o coeficiente de determinação na escala real (sem transformações)).

Todas as equações utilizadas são lineares ou linearizáveis por transformação, de modo que os ajustes foram feitos a partir de retas. No caso de modelos com transformação (geométrica, exponencial e logarítmica), foi feito o retorno para a escala original e, após isso, obtidas as referidas somas de quadrados do resíduo. Os coeficientes de determinação $\left(\mathrm{r}^{2}\right)$ são os obtidos com as variáveis de trabalho $\mathrm{X}$ e $\mathrm{Y}$, caso linear; logaritmo de $\mathrm{Y}$ e logaritmo de $\mathrm{X}$, no caso geométrico; e logaritmo de $\mathrm{Y}$ e $\mathrm{X}$, no caso exponencial. $\mathrm{O}$ número de graus de liberdade é o número de folhas analisadas, menos o número de parâmetros estimados para cada modelo. Para tanto se aplicou o teste F de FisherSnedecor da análise da variância da regressão adotando-se um nível descritivo de significância de 3 \% (MEAD; CURNOW, 1983; NETER; WASSERMAN, 1974). Para isso na escolha do melhor modelo adotou-se os critérios de maior valor para o coeficiente de determinação $\left(\mathrm{r}^{2}\right)$, menor valor para o $\mathrm{p}$ valor do resultado da aplicação do teste $\mathrm{F}$ da análise de variância da regressão.

As análises estatísticas foram realizadas pelo programa estatístico r-projector, R Version 2.13.1 (2011).

\section{RESULTADOS E DISCUSSÃO}

Os valores do comprimento (C) das folhas variaram de 10,0 a $19,0 \mathrm{~cm}$, com valores médios de $13,4 \mathrm{~cm}$. Já a largura (L) máxima das folhas variou de 19,0 a $33,5 \mathrm{~cm}$, com valores médios de $24,8 \mathrm{~cm}$. Para a área foliar real, os valores variaram entre 129,0 e $395,0 \mathrm{~cm}^{2}$, com média de $202,38 \mathrm{~cm}^{2}$ (Tabela 1).

Tabela 1. Valores máximos, mínimos e médios do comprimento ao longo da nervura central, largura e área foliar de 200 limbos de jitirana. UFERSA/Mossoró-RN. 2010.

\begin{tabular}{|c|c|c|c|c|c|c|}
\hline $\begin{array}{c}\text { VARIÁVEL } \\
\text { (CARACTERÍSTICA) }\end{array}$ & $\begin{array}{l}\text { VALOR } \\
\text { MÍNIMO }\end{array}$ & $\begin{array}{l}\text { VALOR } \\
\text { MÁXIMO }\end{array}$ & $\begin{array}{l}\text { VALOR } \\
\text { MÉDIO }\end{array}$ & $\begin{array}{c}\text { VARIÁVEL } \\
\text { (CARACTERÍSTICA) }\end{array}$ & $\begin{array}{l}\text { VALOR } \\
\text { MÍNIMO }\end{array}$ & $\begin{array}{c}\text { VALOR } \\
\text { MÁXIMO }\end{array}$ \\
\hline Comprimento $(\mathrm{cm})$ & 10,0 & 19,0 & 13,4 & $\begin{array}{l}\text { Comprimento } \\
\text { (cm) }\end{array}$ & 10,0 & 19,0 \\
\hline Largura $(\mathrm{cm})$ & 19,0 & 33,5 & 24,8 & Largura $(\mathrm{cm})$ & 19,0 & 33,5 \\
\hline Área foliar $\left(\mathrm{cm}^{2}\right)$ & 129,0 & 395,0 & 202,4 & Área foliar $\left(\mathrm{cm}^{2}\right)$ & 129,0 & 395,0 \\
\hline
\end{tabular}

A distribuição de frequiência percentual do tamanho da área em centímetro quadrado referente aos 200 limbos foliares da jitirana (Merremia aegyptia), em relação as classes de tamanho está descrita na Tabela 2. Pode ser verificado que apenas $1 \%$ da área foliar está compreendida ou relacionada com folhas variando de $129,0 \mathrm{~cm}^{2}$ a $133,2 \mathrm{~cm}^{2}$, enquanto que 95,0 \% da área foliar está relacionada com folhas variando de $133,3 \mathrm{~cm}^{2}$ a $299,0 \mathrm{~cm}^{2}$ indicando que esta espécie vegetal não cultivada possui a maioria de suas folhas com áreas não muito pequenas nem muito grandes, ou seja, áreas relativamente médias, semelhante a resultados encontrados por Pedrinho Junior et al. (2000), Bianco et al. (2002), Pinto et al. (2007) e Duarte et al. (2009). Ainda observando-se os dados dessa tabela, pode-se realizar um estudo descritivo ou uma análise exploratória desses dados, determinando-se estimativas da média, mediana, moda, separatrizes, amplitude total, variância, desvio padrão, erro padrão da média, coeficientes de variação de Pearson e Thorndike, bem como estudar o comportamento dessas variáveis através de um análise gráfica utilizando o histograma e o polígono de frequência, neste caso pode-se descrever toda a biometria dos dados dessa espécie vegetal para posterior análise descritiva e inferencial (SPIEGEL, 2000; COSTA NETO, 2000; SANTANA; RANAL, 2004).

Os resultados da análise de regressão efetuados, relacionando a área foliar real (AR) e as medidas lineares de comprimento (C), largura (L), da soma de $\mathrm{C}+\mathrm{L}$, do produto do comprimento pela largura da folha $\left(\mathrm{C}^{*} \mathrm{~L}\right)$, bem como os ajustes considerando as medidas separadas em uma equação linear múltipla (Tabelas 3 e 4). Observou-se que todas as equações ajustadas permitiram obter estimativas satisfatórias da área foliar de jitirana, com coeficientes de determinação acima de $80 \%$, exceto em poucos casos que ficaram abaixo de $80 \%$, indicando que $80 \%$ das variações observadas na área foliar foram explicadas estatisticamente pelas equações obtidas. As equações que utilizaram o produto entre o comprimento e a largura, passando ou não pela origem, não mostraram diferenças significativas quando comparadas entre si. Isso era esperado, visto que de acordo com Neter e Wasserman (1974), a retirada de uma constante não afeta o comportamento dos dados. 
Tabela 2. Distribuição de frequência percentual de 200 limbos foliares de jitirana, em relação às classes de tamanho em $\mathrm{cm}^{2}$. UFERSA/Mossoró-RN. 2010.

\begin{tabular}{cc}
\hline $\left.\begin{array}{c}\text { Classes } \\
(\text { tamanho em cm }\end{array}\right)$ & $\begin{array}{c}\text { Frequência percentual simples } \\
\left(\mathrm{f}_{\%}\right)\end{array}$ \\
\hline$[100,0---133,3)$ & 1,0 \\
{$[133,3---166,7)$} & 30,5 \\
{$[166,7----200,0)$} & 26,5 \\
{$[200,0----233,3)$} & 16,5 \\
{$[233,3---266,7)$} & 13,0 \\
{$[266,7---300,0)$} & 8,5 \\
{$[300,0---333,3)$} & 2,5 \\
{$[333,3----366,7)$} & 0,5 \\
{$[366,7----400,0)$} & 1,0 \\
\hline
\end{tabular}

Tabela 3. Modelos de regressão de equações lineares e não lineares utilizadas na estimação da área foliar de Jitirana (Merremia aegyptia) em função de medidas lineares do limbo foliar ${ }^{2}$, com o respectivo coeficiente de determinação $\left(\mathrm{r}^{2}\right)$, o valor $\mathrm{P}$ referente à aplicação do teste $\mathrm{F}$ de Fisher - Snedecor ${ }^{1}$ com V $=198$ graus de liberdade, e os estimadores dos parâmetros das equações ajustadas. UFERSA/Mossoró-RN. 2010.

\begin{tabular}{|c|c|c|c|c|}
\hline $\mathrm{ML}_{X_{i}}(\mathrm{~cm})$ & Modelo & $\mathrm{CD}\left(\mathrm{r}^{2}\right)$ & EPER & Teste F (Valor P) \\
\hline $\mathrm{C}$ & $Y=a+b X$ & $89,7 \%$ & $\hat{\boldsymbol{a}}=-289,5 ; \hat{\boldsymbol{b}}=46,6$ & 0,$02160 ; 0,02160$ \\
\hline $\mathrm{C}$ & $Y=a e^{(b X)}$ & $68,2 \%$ & $\hat{\boldsymbol{a}}=52,6 ; \hat{\boldsymbol{b}}=0,1$ & 0,$00001 ; 0,00001$ \\
\hline $\mathrm{C}$ & $Y=a+b \ln (X)$ & $88,0 \%$ & $\hat{\boldsymbol{a}}=-1285,2 ; \hat{\boldsymbol{b}}=626,3$ & 0,$00940 ; 0,00940$ \\
\hline $\mathrm{C}$ & $Y=a X^{b}$ & $68,1 \%$ & $\hat{\boldsymbol{a}}=2,8 ; \hat{\boldsymbol{b}}=1,8$ & 0,$00001 ; 0,00001$ \\
\hline $\mathrm{L}$ & $Y=a+b X$ & $87,1 \%$ & $\hat{\boldsymbol{a}}=-334,1 ; \hat{\boldsymbol{b}}=27,2$ & 0,$00590 ; 0,00590$ \\
\hline $\mathrm{L}$ & $Y=a e^{(b X)}$ & $64,6 \%$ & $\hat{\boldsymbol{a}}=47,2 ; \hat{\boldsymbol{b}}=0,1$ & 0,$00001 ; 0,00001$ \\
\hline $\mathrm{L}$ & $Y=a+b \ln (X)$ & $85,6 \%$ & $\hat{\boldsymbol{a}}=-1814,6 ; \hat{\boldsymbol{b}}=671,4$ & 0,$00270 ; 0,00270$ \\
\hline $\mathrm{L}$ & $Y=a X^{b}$ & $64,4 \%$ & $\hat{\boldsymbol{a}}=0,6 ; \hat{\boldsymbol{b}}=1,9$ & 0,$00001 ; 0,00001$ \\
\hline$(\mathrm{C} * \mathrm{~L})$ & $Y=b X$ & $97,1 \%$ & $\hat{\boldsymbol{b}}=0,5$ & 0,$00001 ; 0,00001$ \\
\hline$(\mathrm{C} * \mathrm{~L})$ & $Y=a+b X$ & $87,1 \%$ & $\hat{\boldsymbol{a}}=-334,1 ; \hat{\boldsymbol{b}}=27,1$ & 0,$00590 ; 0,00590$ \\
\hline$(\mathrm{C} * \mathrm{~L})$ & $Y=a e^{(b X)}$ & $64,6 \%$ & $\hat{\boldsymbol{a}}=47,2 ; \hat{\boldsymbol{b}}=0,1$ & 0,$00001 ; 0,00001$ \\
\hline$(\mathrm{C} * \mathrm{~L})$ & $Y=a+b \ln (X)$ & $85,6 \%$ & $\hat{a}=-1814,6 ; \hat{b}=671,4$ & 0,$00270 ; 0,00270$ \\
\hline$(\mathrm{C} * \mathrm{~L})$ & $Y=a X^{b}$ & $64,4 \%$ & $\hat{\boldsymbol{a}}=0,6 ; \hat{\boldsymbol{b}}=1,9$ & 0,$00001 ; 0,00001$ \\
\hline$(\mathrm{C}+\mathrm{L})$ & $Y=a+b X$ & $93,0 \%$ & $\hat{\boldsymbol{a}}=-353,9 ; \hat{\boldsymbol{b}}=18,1$ & 0,$09710 ; 0,09710$ \\
\hline$(\mathrm{C}+\mathrm{L})$ & $Y=a e^{(b X)}$ & $70,0 \%$ & $\hat{\boldsymbol{a}}=44,2 ; \hat{\boldsymbol{b}}=0,1$ & 0,$00001 ; 0,00001$ \\
\hline$(\mathrm{C}+\mathrm{L})$ & $Y=a+b \ln (X)$ & $91,4 \%$ & $\hat{\boldsymbol{a}}=-2181,3 ; \hat{\boldsymbol{b}}=692,3$ & 0,$04720 ; 0,04720$ \\
\hline$(\mathrm{C}+\mathrm{L})$ & $Y=a X^{b}$ & $69,5 \%$ & $\hat{\boldsymbol{a}}=0,2 ; \hat{\boldsymbol{b}}=2,0$ & 0,$00001 ; 0,00001$ \\
\hline$(\mathrm{C}+\mathrm{L})$ & $Y=a+b X$ & $93,0 \%$ & $\hat{\boldsymbol{a}}=-353,9 ; \hat{\boldsymbol{b}}=18,1$ & 0,$09710 ; 0,09710$ \\
\hline
\end{tabular}

ML - Modelo linear; CD - Coeficiente de determinação; EPER - Estimativas dos parâmetros da equação de regressão; C - comprimento; L - largura; NS Não significativo (valor p superior a 0,05 ); * - Estatisticamente significativo (valor inferior a 0,05 ). 
Tabela 4. Modelos de regressão de equações lineares múltiplas utilizadas na estimação da área foliar de Jitirana (Merremia aegyptia) em função de medidas lineares do limbo foliar ${ }^{2}$, com o respectivo coeficiente de determinação $\left(\mathrm{r}^{2}\right)$, o valor $\mathrm{P}$ referente a aplicação do teste $\mathrm{F}$ de Fisher - Snedecor ${ }^{1}$ com V = 198 graus de liberdade, e os estimadores dos parâmetros das equações ajustadas. UFERSA/Mossoró-RN. 2010.

\begin{tabular}{|c|c|c|c|c|}
\hline ML $X_{i}(\mathrm{~cm})$ & Modelo & $\mathrm{CD}\left(\mathrm{r}^{2}\right)$ & EPER & Teste F (Valor P) \\
\hline$(\mathrm{C} ; \mathrm{L})$ & $Y=a+b_{1} X_{1}+b_{2} X_{2}$ & $94,0 \%$ & $\hat{a}=-346,9 ; \hat{b}_{1}=27,6 ; \hat{b}_{2}=12,6$ & $\begin{array}{c}0,00001 ; 0,00001 \mathrm{e} \\
0,00001\end{array}$ \\
\hline$\left[\left(\mathrm{C}^{*} \mathrm{~L}\right) ;(\mathrm{C}+\mathrm{L})\right]$ & $Y=a+b_{1} X_{1}+b_{2} X_{2}$ & $94,5 \%$ & $\hat{a}=76,2 ; \hat{b}_{1}=1,2 ; \hat{b}_{2}=-4,0$ & $\begin{array}{c}0,20970 ; 0,00010 \mathrm{e} \\
0,18850\end{array}$ \\
\hline$[(\mathrm{C}) ;(\mathrm{C} * \mathrm{~L})]$ & $Y=a+b_{1} X_{1}+b_{2} X_{2}$ & $94,4 \%$ & $\hat{a}=-20,6 ; \hat{b}_{1}=2,7 ; \hat{b}_{2}=0,9$ & $\begin{array}{c}0,38700 ; 0,45220 \mathrm{e} \\
0,00010\end{array}$ \\
\hline$[(\mathrm{L}) ;(\mathrm{C} * \mathrm{~L})]$ & $Y=a+b_{1} X_{1}+b_{2} X_{2}$ & $94,5 \%$ & $\hat{\boldsymbol{a}}=26,9 ; \hat{\boldsymbol{b}}_{\mathbf{1}}=-2,3 ; \hat{\boldsymbol{b}}_{2}=1,09$ & 0,$2936 ; 0,22500$ e 0,00010 \\
\hline$\left[(\mathrm{C}) ;\left(\mathrm{C}^{2}\right)\right]$ & $Y=a+b_{1} X+b_{2} X^{2}$ & $88,1 \%$ & $\hat{\boldsymbol{a}}=17,3 ; \hat{\boldsymbol{b}}_{\mathbf{1}}=0,1 ; \hat{\boldsymbol{b}}_{\mathbf{2}}=0,00001$ & $\begin{array}{c}0,00010 ; 0,01360 \mathrm{e} \\
0,00010\end{array}$ \\
\hline$\left[(\mathrm{L}) ;\left(\mathrm{L}^{2}\right)\right]$ & $Y=a+b_{1} X+b_{2} X^{2}$ & $90,1 \%$ & $\hat{\boldsymbol{a}}=8,6 ; \hat{\boldsymbol{b}}_{\mathbf{1}}=0,009 ; \hat{\boldsymbol{b}}_{\mathbf{2}}=0,00001$ & $\begin{array}{c}0,00010 ; 0,00060 \mathrm{e} \\
0,00010\end{array}$ \\
\hline
\end{tabular}

NS - Não significativo (valor p superior a 0,05 ); * Estatisticamente significativo (valor inferior a 0,05 ).

Os maiores valores do coeficiente de determinação e os menores valores da soma de quadrados do resíduo foram observados para as regressões lineares simples entre a área real e o comprimento, entre a área real e a largura, linear simples passando pela origem entre a área real e o produto comprimento com largura e linear múltipla entre a área real com o comprimento e a largura, indicando ser as equações que permitiram obter estimativas mais acuradas da área foliar da jitirana (Merremia aegyptia) (Tabelas 3 e 4). Nota-se também que essas equações apresentaram estimativas do coeficiente de determinação de $89,7 \%, 87,15,97,1 \%$ e $94,0 \%$, respectivamente, significando que, da variabilidade total existente na área foliar, $89,7 \%, 87,15,97,1 \%$ e 94,0\% podem ser explicadas pelas equações de regressões lineares simples entre a área real e o comprimento, entre a área real e a largura, linear simples passando pela origem entre a área real e o produto comprimento com largura e linear múltipla entre a área real com o comprimento e a largura, respectivamente.

A equação linear simples com a reta passando pela origem $(A R=0,5(C * L))$ é a mais recomendada para este fim, pois não altera expressivamente a soma de quadrados do resíduo e é de fácil utilização do ponto de vista prático, cujo coeficiente de determinação correspondeu a 97,1\%, mostrando também desse modo que o resultado traduz um elevado poder explicativo dessa equação no que se refere a estimação da área foliar dessa espécie vegetal. Também a estimativa da área foliar da jitirana (Merremia aegyptia), pode ser feita pelas equações $\mathrm{AR}=-289,5+46,6 \mathrm{C}, \mathrm{AR}=-$ $334,1+27,2 \mathrm{~L}$ e AR $=-346,9+27,6 \mathrm{C}+12,6 \mathrm{~L}$.

Vale ressaltar que as escolhas dos modelos foram feitos com parcimônia além, é claro, dos critérios estatísticos do coeficiente de determinação e do valor p do teste de hipótese de Fisher - Snedecor da análise de variância da regressão (MEAD; CURNOW, 1983; NETER; WASSERMAN, 1974). Resultados semelhantes ao nosso trabalho foram obtidos por alguns autores (BIANCO et al., 2003; BIANCO; PITELLI, 2002; BIANCO et al., 2004; TOFOLI et al., 1998).

\section{CONCLUSÕES}

A jitirana (Merremia aegyptia) apresentou valores médios de comprimento das folhas, largura e área foliar real iguais a $13,5 \mathrm{~cm}, 28,8 \mathrm{~cm}$ e $202,38 \mathrm{~cm}^{2}$, respectivamente.

95\% da área foliar de 200 limbos está relacionada com folhas de tamanho variando de $133,3 \mathrm{~cm}^{2}$ a $299,0 \mathrm{~cm}^{2}$.

As melhores equações para estimação da área foliar da jitirana em ordem de importância foram: modelo linear simples passando pela origem em função do produto do comprimento com a largura da folha; linear simples em função do comprimento; linear simples em função da largura; e o modelo de regressão linear múltipla, modelada em função do comprimento e largura simultaneamente.

\section{AGRADECIMENTOS}

Ao Grupo de Pesquisa Jitirana, comprometido com o estudo de espécies espontâneas da caatinga \{jitirana (Merremia aegyptia); flor-de-seda (Calotropis procera) e mata-pasto (Senna uniflora e Senna Obtusifolia)\} e à UFERSA - Mossoró/RN, por oferecerem aparato físico para o desenvolvimento dos trabalhos.

\section{REFERÊNCIAS BIBLIOGRÁFICAS}

BIANCO, S.; BIANCO, M. S.; PAVANI, M. C. M. D. and DUARTE, D.J.. Estimativa da área foliar de Ipomoea hederifolia e Ipomoea nil Roth: usando dimensões lineares do limbo foliar. Revista Planta daninha. 2007, v. 25, n.2, p. 325-329.

BIANCO, S.; CARVALHO, L. B.; BIANCO, M. S. Estimativa da área foliar de Sida cordifolia e Sida rhombifolia usando dimensões lineares do limbo foliar. Revista Planta Daninha, Viçosa, v. 26, n. 4, p. 807-813, 2008. 
BIANCO, S.; PITELli, R. A.; CARVALHO, L. B. Estimativa da área foliar de Cissampelos glaberrima L. usando dimensões lineares do limbo foliar. Revista Planta Daninha, Viçosa, v. 20, n. 3, p. 353-356, 2002.

BIANCO, S.; PITELli, R. A.; CARVALHO, L. B. Estimativa da área foliar de Tridax procumbens usando dimensões lineares do limbo foliar. Revista Planta Daninha, Viçosa, v. 22, n. 2, p. 247-250, 2004.

BIANCO, S.; PITELli, R. A.; PAVANI, M. C. M. D.; SILVA, R. C. Estimativa de área foliar de plantas daninhas. XIII - Amaranthus retroflexus L. Revista Ecossistema, Espírito Santo do Pinhal, v. 20, p. 5-9, 1995.

BIANCO, S.; PITELLI, R. A.; PERECIN, D. Métodos para estimativa de foliar de plantas daninhas 2. Wissadula subpeltata (Kuntze) Fries. Revista Planta Daninha, Viçosa, v.6, n.1, p. 21-24, 1983.

BIANCO, S.; PITELLI, R. A.; PITELLI, A. M. C. M. Estimativa da área foliar de Typha latifolia L. usando dimensões lineares do limbo foliar. Revista Planta Daninha, Viçosa, v. 21, n. 2, p. 257-261, 2003.

CARMO FILHO, F. do; ESPÍNOLA SOBRINHO, J.; MAIA NETO, J.M. Dados climatológicos de Mossoró: um município semi-árido nordestino. Mossoró: ESAM, 1991, 121p. (Coleção mossoroense, série C, 30).

CORRÊIA, M. P. Dicionário das plantas úteis do Brasil e das Exóticas cultivadas. 4. ed. Brasília: Ministério da Agricultura, 1984. 765p.

COSTA NETO, P. L. O. Estatística. São Paulo: Edgard Blücher, 2000. 264p.

DUARTE, D. J.; BIANCO, S.; LEONARDO, B. C.; PANOSSO, A. R. Estimativa da área foliar de Euphorbia heterophylla. Revista Planta Daninha, Viçosa, v. 27, n. 3, p. 527-531, 2009.

KMENTA, J. Elementos de econometria: teoria econométrica básica. 2. ed. São Paulo: ATLAS, 1990. 469 p.

LINHARES, P. C. F.; LEITE DE LIMA, G. K.; MADALENA. J. A. da S.; MARACAJÁ, P. B.; FERNANDES, P. L. de O. Adição de jitirana ao solo no desempenho de rúcula cv. Folha Larga. Revista Caatinga, v.21, n.5, p.89-94, 2008.

LINHARES, P.C.F.; PEREIRA, M.F.S.; DIAS, M.A.V.; HOLANDA, A.K.B.; MOREIRA, J.C. Rendimento de coentro (CoriandrumsativumL.) em sistema de adubação verde com a planta jitirana (MerremiaaegyptiaL.). Revista Brasileira Plantas Medicinais., Botucatu, v.14, n.esp., p.143-148, 2012.

MARSHALL, J. K. Methods of leaf area measurement of large and small leaf samples. Photosynthetica, $\mathrm{Na}$ Karlovce La, Republica Checa, v. 2, p. 41-47, 1968.

MEAD, R.; CURNOW, R. N. Statistical methods in agriculture and experimental biology. New York: Chapman and Hall, 1983. 335 p.
NETER, J.; WASSERMAN, W. Applied models: Regressions, analysis of variance and experimental designs. Illinois: Rechard D. Irwin, 1974. 842 p.

PEDRINHO JÚNIOR, A. F. F.; BARBOSA JUNIOR, A. de F.; BIANCO, S.; PITELLI, R. A. Estimativa da área foliar de plantas daninhas: Portulaca oleracea L. Revista Ecossistema, Espírito Santo do Pinhal, v. 25, n.1, p. 8688,2000 .

PEDRO JUNIOR, M. J.; RIBEIRO, I. J. A.; MARTINS, F. P. Determinação da área foliar em videira cultivar Niagara rosada. Revista Bragantia, Campinas, v. 45, n. 1, p. 199204, 1986.

PERESSIN, V. A.; PITELLI, R. A.; PERECIN, D. Métodos para estimativa da área foliar de plantas daninhas. Planta Daninha, Viçosa, v. 7, n. 2, p. 48-52, 1984.

PINTO, M. S. C.; ANDRADE, A. P.; PEREIRA, W. E.; ARRUDA, F. P.; ANDRADE, M. V. M. Modelo para estimativa da área foliar da maniçoba. Revista Ciências Agronômica, Fortaleza, v. 38, n. 4, p. 391-395, 2007.

R VERSION 2.13.1, Viena, Aústria: Foundation for Estatistical Computing, 2011 (Software).

ROSSETO, R. R.; PITELli, R. L. C. M.; PITELli, R. A. Estimativa da área foliar de plantas daninhas: PoaiaBranca. Revista Planta Daninha, Viçosa, v. 15, n. 1, p. 2529, 1997.

SANTANA, D. G.; RANAL, M. A. Análise da germinação: um enfoque estatístico. Brasília: Editora UNB, 2004. 54 p.

SILVA, L. C.; SANTOS, J. W. dos; VIEIRA, D. J.; BELTRÃO, N. E. de M.; ALVES, I.; JERÔNIMO, J. F. Um método simples para se estimar área foliar de plantas de gergelim (Sesamum indicum L.). Revista Brasileira de Oleaginosas e Fibrosas, Campina Grande, v. 6, n. 1, p. 491-496, 2002

SILVA, N. F. da; FERREIRA, F. A.; FONTES, P. C. R.; CARDOSO, A. A. Modelos para estimar a área foliar de abóbora por meio de medidas lineares. Revista Ceres, Viçosa, v. 45, n. 259, p. 287-291, 1998.

SOUZA LIMA, C. J. G. de; OLIVEIRA, F. A. de; MEDEIROS, J. F. de; OLIVEIRA, M. K. T. de; OLIVEIRA FILHO, A. F. de; Modelos matemáticos para estimativa de área foliar de feijão caupi. Revista Caatinga, Mossoró, v. 21, n. 1, p. 121-127, 2008.

SOUZA NETO, A. G. Avaliação da área foliar de cinco espécies florestais ocorrentes no semiárido paraibano. 2009. 37f. Monografia (Graduação em Engenharia Florestal) - Universidade Federal de Campina Grande. Patos, 2009.

SPIEGEL, M. R. Estatística. São Paulo: Macron Books, 4. ed. 2000. 580p.

TOFOLI, G. R.; BIANCO, S.; PAVANI, M. C. M. D. Estimativa da área foliar de Solanum americanum Mill. Revista Planta Daninha, Viçosa, v. 16, n. 2, p. 149-152, 1998. 